\title{
Propagation characteristics of nighttime mesospheric and thermospheric waves observed by optical mesosphere thermosphere imagers at middle and low latitudes
}

\author{
K. Shiokawa, Y. Otsuka, and T. Ogawa \\ Solar-Terrestrial Environment Laboratory, Nagoya University, Toyokawa 442-8507, Japan \\ (Received September 20, 2007; Revised April 21, 2008; Accepted April 22, 2008; Online published May 14, 2009)
}

\begin{abstract}
We review measurements of nighttime atmospheric/ionospheric waves in the upper atmosphere in Japan, Indonesia, and Australia, using all-sky airglow imagers of optical mesosphere thermosphere imagers (OMTIs). The imagers observe two-dimensional patterns of airglow emissions from oxygen (wavelength: $557.7 \mathrm{~nm}$ ) and hydroxyl $(\mathrm{OH})$ (near-infrared band) in the mesopause region $(80-100 \mathrm{~km})$ and from oxygen $(630.0 \mathrm{~nm})$ in the thermosphere/ionosphere $(200-300 \mathrm{~km})$. Several statistical studies were done to investigate propagation characteristics of small-scale (less than $100 \mathrm{~km}$ ) gravity waves in the mesopause region and medium-scale traveling ionospheric disturbances (MSTIDs, $\sim 100-1,000 \mathrm{~km}$ ) in the thermosphere/ionosphere. Clear seasonal variations of occurrence and propagation directions were reported for these waves. The propagation directions in the mesopause region are controlled by wind filtering, ducting processes and relative location to the wave sources in the troposphere. Poleward-propagating waves tend to be observed in the summer in the mesopause region at several stations, suggesting that mesospheric gravity waves are generated by intense convective activity in the equatorial troposphere. On the other hand, systematic equatorward and westward motions were observed for all seasons for nighttime MSTIDs in the midlatitude ionosphere with geomagnetic conjugacy between the northern and southern hemispheres. Ionospheric instabilities may play important role for the generation and propagation of these MSTIDs. We also give an example of simultaneous observation of quasi-periodic southward-moving waves in the mesopause region and in the thermosphere at the geographic equator. From these results, we discuss mean wind acceleration by mesospheric gravity waves and penetration of gravity waves from the mesosphere to the thermosphere.
\end{abstract}

Key words: Gravity wave, airglow image, mesopause region, thermosphere, ionosphere, MSTID.

\section{Introduction}

During nighttime, Earth's atmosphere at high altitudes (above $80 \mathrm{~km}$ ) emits weak (invisible) light at many line wavelengths, called airglow emissions. The major airglow emissions are two lines at wavelengths of 557.7 and $630.0 \mathrm{~nm}$ from atomic oxygen and many lines from hydroxyl $(\mathrm{OH})$ at wavelengths in the near-infrared region.

The oxygen green-line emission at a wavelength of $557.7 \mathrm{~nm}$ and the OH-band near-infrared emissions have emitting layers near the mesopause region $(\sim 95 \pm 5 \mathrm{~km}$ and $\sim 86 \pm 5 \mathrm{~km}$, respectively), where the atmospheric temperature is lowest in Earth's atmosphere. Since the electron density is very low at this altitude range, the variations of these airglow emissions are controlled by variations in densities and temperatures in the neutral atmosphere, mostly due to atmospheric gravity waves.

The emission of the oxygen red line at a wavelength of $630.0 \mathrm{~nm}$ comes from the lower thermosphere at altitudes of 200-300 km. This emission is basically a result of excitation of atomic oxygen by the interaction between molecular oxygen $\left(\mathrm{O}_{2}\right)$ and the oxygen ion $\left(\mathrm{O}^{+}\right)$(e.g., Sobral et

Copyright (c) The Society of Geomagnetism and Earth, Planetary and Space Sciences (SGEPSS); The Seismological Society of Japan; The Volcanological Society of Japan; The Geodetic Society of Japan; The Japanese Society for Planetary Sciences; TERRAPUB. al., 1993). The $\mathrm{O}^{+}$density is basically equal to the electron density in the ionospheric $F$-layer. Thus, the intensity of the 630.0-nm airglow is a sensitive indicator of the variations of ionospheric ion density and the height of the ionosphere.

Using highly sensitive all-sky imagers with a cooled charge-coupled device (CCD) and narrow band-pass filters, one can obtain two-dimensional images of airglow emissions and hence the atmospheric and ionospheric waves at these altitude ranges (e.g., Taylor et al., 1995; Mendillo et al., 1997). Since the development of cooled-CCD camera in the 1990s, there have been a lot of imaging measurements of both mesospheric and thermospheric waves through airglow emissions. However, the observed wave characteristics at different latitudes have not yet been compared. In this paper, we review the wave characteristics obtained in the Japanese longitudinal sector, mainly by the airglow imagers that belong to optical mesosphere thermosphere imagers (OMTIs). The wave characteristics, particularly the propagation directions of the waves, show clear seasonal and latitudinal dependences. We also give an example of waves observed simultaneously in the mesopause region and in the thermosphere and discuss the penetration of gravity waves from the mesosphere to the thermosphere. 


\section{Details of the Airglow Imagers}

OMTIs have made airglow imaging observations since 1997 (Shiokawa et al., 1999). OMTIs consist of all-sky cooled-CCD imagers, a Fabry-Perot interferometer, meridian scanning photometers, and airglow temperature photometers. In this paper, we focus on the results obtained by the all-sky imagers. The imagers use thinned and backilluminated cooled CCDs made by Hamamatsu Photonics with $512 \times 512$ pixels. For details of the imagers, see Shiokawa et al. (2000a).

Table 1 lists filters and sensitivities of the imagers of OMTIs. All 11 airglow imagers of OMTIs have at least four filters on rotating wheels to measure 557.7-nm (channel 1), 630.0-nm (channel 2), and OH-band (channel 3) emissions, and sky background emissions at $572.5 \mathrm{~nm}$ (channel 5). Some imagers have additional filters to measure emissions from thermospheric oxygen $(777.4 \mathrm{~nm})$, mesospheric sodium $(589.3 \mathrm{~nm})$, auroral oxygen $(844.6 \mathrm{~nm})$, and hydrogen $(486.1 \mathrm{~nm})$ at channels 4 and 6-7. The bandwidths of the narrow band-pass filters for the measurement of line emissions are $\sim 1-2 \mathrm{~nm}$. The exposure times to obtain $557.7 \mathrm{~nm}, 630.0 \mathrm{~nm}$, and $\mathrm{OH}$-band images are $105 \mathrm{~s}$, $165 \mathrm{~s}$, and $10-15 \mathrm{~s}$, respectively. Imagers 6,7 , and 9 are operated with much shorter exposure times by using $2 \times 2$ binning of the CCD pixel, to obtain images in faster time resolutions for measurement of auroras at high latitudes. In the routine operation of the imagers, the images of channels $1-3(557.7 \mathrm{~nm}, 630.0 \mathrm{~nm}$, and $\mathrm{OH}$ band) are sequentially obtained, while images of other channels are taken intermittently. The time resolutions of channels 1-3 are $\sim 5.5 \mathrm{~min}$ for the case of no binning and $\sim 2$ min with 2 $\times 2$ binning.

The sensitivities of the imagers are $\sim 0.03-0.4$ counts/Rayleighs/s, giving 300-4,000 counts for a $100 \mathrm{R}$ airglow emission with a 100-s exposure without pixel binning. The sensitivity becomes better for later imagers (larger imager numbers), indicating improvement of the quantum efficiency of the CCD. The sensitivities in unit of count/R/s of imagers with 16-bit A/D converters of CCDs are $\sim 4$ times higher than those with 14-bit A/D converters, because the factor to convert electrons to counts is 4 times larger for 16-bit A/D converters. However, the dark and read-out noises in unit of count also become 4 times larger for the 16-bit CCDs. The typical dark and read-out noises of recent CCDs by Hamamatsu Photonics (C9299-02) are 0.8 electrons/pixel/s and 8 electrons root-mean-square (r.m.s.), respectively. The factors to convert from electrons on the CCD pixel to the output counts are $\sim 0.8$ and $\sim 0.2$ electrons/counts for 14-bit and 16-bit A/D converters, respectively.

The calibration of all imagers to obtain these sensitivities was done by using a 2-m integrating sphere and a grating spectrometer at the National Institute of Polar Research, Japan. The details of the calibration were reported by Shiokawa et al. (2000a). We calibrated the imagers 1 and 4 several times during the time they were repaired. From these multiple calibrations, we could identify the degradation of imager sensitivity due to long-term measurements. The degradation was mainly caused by Mamiya 24$\mathrm{mm} / \mathrm{F} 5.6$ fish-eye lenses, which were used at the front op-

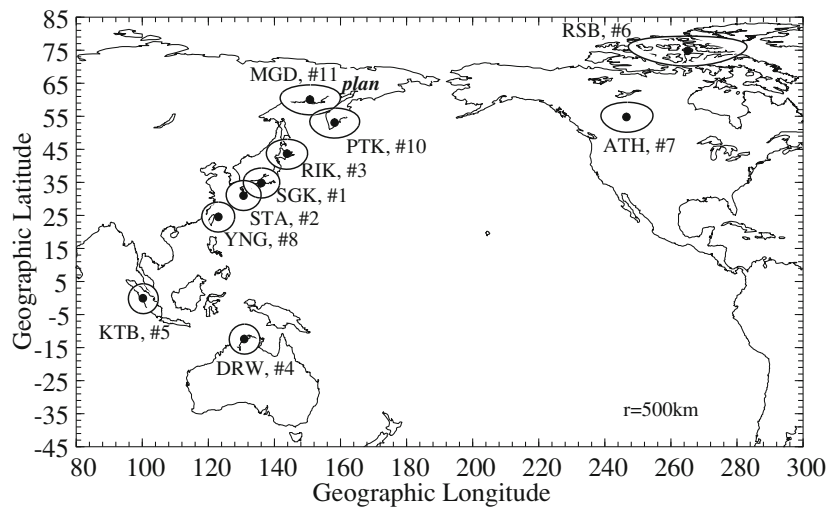

Fig. 1. Map showing the current and planned stations of all-sky cooled-CCD imagers of OMTIs. The imager numbers installed at these stations are also indicated. The station names are Magadan (MGD), Paratunka (PTK), Rikubetsu (RIK), Shigaraki (SGK), Sata (STA), Yonaguni (YNG), Kototabang (KTB), Darwin (DRW), Resolute Bay (RSB), and Athabasca (ATH). The imager at YNG belongs to the Electronic Navigation Research Institute (ENRI), Japan.

tics. Long-term exposure of the Mamiya lens to daytime UV sunlight causes degradation of the transmission of the lens, which is recognizable because the lens surface turns white. For example, the sensitivity of imager 4 degraded down to $\sim 70-90 \%$ after $\sim 5.5$ years of routine operation at Darwin $\left(12.4^{\circ} \mathrm{S}, 131.0^{\circ} \mathrm{E}\right)$, Australia, in the tropical climate. By replacing the fish-eye lens with a new one, the sensitivity recovered to the initial values. This fact indicates that the degradations of filter transmission and quantum efficiency of CCDs were not significant. We did measure the filter transmission functions at these multiple calibrations and confirmed that the filter transmissions were not significantly changed. For some cases, however, we found defects around the edge of the band-pass filters after the long-term operation. Such defects may be caused by invasion of humidity from the edge of the filters. In the case of imager 1 , the CCD repair on October, 2006, seems to increase the sensitivity, although the degradation due to the fish-eye lens was clear after $\sim 9$ years of operation.

Figure 1 shows the current and planned stations of all-sky imagers of OMTIs (The imager at Yonaguni (YNG) belongs to the Electronic Navigation Research Institute (ENRI)). Table 2 lists the latitudes and longitudes of the stations. Imager 6 at Resolute Bay (RSB) observes sun-aligned auroral arcs and polar cap patches (Hosokawa et al., 2006). Imager 7 at Athabasca (ATH) observes auroras at subauroral latitudes (e.g., Sakaguchi et al., 2007). Other imagers at mid- and low latitudes in Japan (Rikubetsu (RIK), Shigaraki (SGK), Sata (STA), and Yonaguni (YNG)), Indonesia (Kototabang (KTB)), and Australia (Darwin (DRW)) observe atmospheric gravity waves in the mesopause region and traveling ionospheric disturbances and plasma bubbles in the thermosphere and the ionosphere. We plan to install one more imager (imager 11) in Magadan (MGD) in 2008, in collaboration with the Institute of Cosmophysical Research and Radiowave Propagation (IKIR), the Far Eastern Branch of the Russian Academy of Sciences. Imager 9 used to be operated at Ithaca, NY, $\left(42.5^{\circ} \mathrm{N}, 283.6^{\circ} \mathrm{E}\right)$ for June $2006-$ April 2007, and then was used/stored for campaign obser- 
Table 1. Filter details, typical exposure times, and sensitivities for all-sky nightglow imagers. The sensitivities are those at (256, 256) pixels (near the image center).

\begin{tabular}{|c|c|c|c|c|c|c|c|c|}
\hline Ch. & Emission & $\begin{array}{c}\text { Wavelength } \\
(\mathrm{nm})\end{array}$ & $\begin{array}{c}\text { Bandwidth } \\
(\mathrm{nm})\end{array}$ & $\begin{array}{c}\text { Exposure } \\
(\mathrm{sec})\end{array}$ & & $\begin{array}{l}\text { Sensitivity } \\
\text { (count/R/s) }\end{array}$ & & \\
\hline \multicolumn{9}{|c|}{1 (14 bit), Shigaraki } \\
\hline & & & & & $* 1$ & $* 2$ & $* 3$ & $* 4$ \\
\hline 1 & OI & 557.7 & 1.76 & 105 & 0.029 & 0.031 & 0.053 & 0.051 \\
\hline 2 & OI & 630.0 & 1.90 & 165 & 0.038 & 0.040 & 0.061 & 0.062 \\
\hline 3 & $\mathrm{OH}$ & $720-910^{*}$ & 190 & 15 & - & - & - & - \\
\hline 4 & $\mathrm{Na}$ & 589.3 & 1.83 & 165 & 0.032 & 0.034 & 0.054 & 0.055 \\
\hline 5 & bg & 572.5 & 1.75 & 105 & 0.031 & 0.035 & 0.057 & 0.057 \\
\hline \multicolumn{9}{|c|}{ *1: Jan. 08, 1998, initial calibration } \\
\hline \multicolumn{9}{|c|}{$* 2:$ Oct. 26,2006 , with old fish-eye lens (after repair of CCD) } \\
\hline \multicolumn{9}{|c|}{ *3: Oct. 26, 2006, with new fish-eye lens } \\
\hline \multicolumn{9}{|c|}{ *4: Feb. 21, 2007, with new CCD transfer level } \\
\hline \multicolumn{9}{|c|}{ Imager 2 (14 bit), Sata } \\
\hline 1 & OI & 557.7 & 1.85 & 105 & 0.044 & & & \\
\hline 2 & OI & 630.0 & 1.94 & 165 & 0.055 & & & \\
\hline 3 & $\mathrm{OH}$ & $720-910^{*}$ & 190 & 15 & - & & & \\
\hline 4 & $\mathrm{O} 2$ & 865.0 & 10 & 105 & - & & & \\
\hline 5 & bg & 572.5 & 1.95 & 105 & 0.050 & & & \\
\hline \multicolumn{9}{|c|}{ Imager 3 (14 bit), Rikubetsu } \\
\hline 1 & OI & 557.7 & 1.78 & 105 & 0.049 & & & \\
\hline 2 & OI & 630.0 & 1.82 & 165 & 0.053 & & & \\
\hline 3 & $\mathrm{OH}$ & $720-910^{*}$ & 190 & 15 & - & & & \\
\hline 4 & $\mathrm{H} \beta$ & 486.1 & 1.92 & - & 0.005 (at $465.1 \mathrm{~nm})$ & & & \\
\hline 5 & bg & 572.5 & 1.77 & 105 & 0.050 & & & \\
\hline \multicolumn{9}{|c|}{ Imager 4 (14 bit), Darwin } \\
\hline & & & & & $* 1$ & $* 2$ & $* 3$ & \\
\hline 1 & OI & 557.7 & 2.04 & 105 & 0.110 & 0.0839 & 0.105 & \\
\hline 2 & OI & 630.0 & 2.02 & 165 & 0.116 & 0.101 & 0.115 & \\
\hline 3 & $\mathrm{OH}$ & $720-910^{*}$ & 190 & 10 & - & - & - & \\
\hline 4 & OI & 777.4 & 1.94 & 165 & 0.074 & 0.0645 & 0.0718 & \\
\hline 5 & bg & 572.5 & 2.04 & 105 & 0.118 & 0.0990 & 0.122 & \\
\hline \multicolumn{9}{|c|}{$* 1:$ Dec. 12,2000 , initial calibration } \\
\hline \multicolumn{9}{|c|}{ *2: Apr. 25, 2006, with old fish-eye lens } \\
\hline \multicolumn{9}{|c|}{ *3: Apr. 25,2006 , with new fish-eye lens } \\
\hline \multicolumn{9}{|c|}{ Imager 5 (14 bit), Kototabang } \\
\hline 1 & OI & 557.7 & 1.96 & 105 & 0.056 & & & \\
\hline 2 & OI & 630.0 & 1.98 & 105 & 0.115 & & & \\
\hline 3 & $\mathrm{OH}$ & $720-910^{*}$ & 190 & 15 & - & & & \\
\hline 4 & OI & 777.4 & 1.78 & 165 & 0.089 & & & \\
\hline 5 & bg & 572.5 & 2.06 & 105 & 0.082 & & & \\
\hline \multicolumn{9}{|c|}{ Imager 6 (14 bit), Resolute Bay } \\
\hline 1 & OI & 557.7 & 1.90 & 30 & 0.082 & & & \\
\hline 2 & OI & 630.0 & 1.87 & 30 & 0.100 & & & \\
\hline 3 & $\mathrm{OH}$ & $720-910$ & 190 & 2 & - & & & \\
\hline 4 & OI & 777.4 & 1.86 & 45 & 0.080 & & & \\
\hline 5 & bg & 572.5 & 1.64 & 30 & 0.092 & & & \\
\hline 6 & $\mathrm{Na}$ & 589.3 & 1.82 & 30 & 0.109 & & & \\
\hline \multicolumn{9}{|c|}{ Imager 7 (16 bit), Athabasca } \\
\hline 1 & OI & 557.7 & 1.76 & 5 & 0.246 & & & \\
\hline 2 & OI & 630.0 & 1.64 & 30 & 0.369 & & & \\
\hline 3 & $\mathrm{OH}$ & $720-910$ & 190 & 1 & - & & & \\
\hline 4 & $\mathrm{H} \beta$ & 486.1 & 1.32 & 40 & $0.254($ at $486.1 \mathrm{~nm})$ & & & \\
\hline 5 & bg & 572.5 & 1.69 & 15 & 0.262 & & & \\
\hline 6 & OI & 844.6 & 1.30 & 25 & 0.177 & & & \\
\hline 7 & $\mathrm{Na}$ & 589.3 & 1.56 & 15 & 0.484 & & & \\
\hline
\end{tabular}

*Band pass at $720-910 \mathrm{~nm}$ with a notch at $865 \mathrm{~nm}$ to suppress the $\mathrm{O}_{2}(0,1)$ emission. 
Table 1. (continued).

\begin{tabular}{|c|c|c|c|c|c|}
\hline Ch. & Emission & $\begin{array}{c}\text { Wavelength } \\
(\mathrm{nm})\end{array}$ & $\begin{array}{c}\text { Bandwidth } \\
(\mathrm{nm})\end{array}$ & $\begin{array}{c}\text { Exposure } \\
(\mathrm{sec})\end{array}$ & $\begin{array}{l}\text { Sensitivity } \\
\text { (count/R/s) }\end{array}$ \\
\hline \multicolumn{6}{|c|}{ Imager 8 (16 bit), Yonaguni } \\
\hline 1 & OI & 557.7 & 1.69 & 105 & 0.360 \\
\hline 2 & OI & 630.0 & 1.56 & 165 & 0.498 \\
\hline 3 & $\mathrm{OH}$ & $720-910$ & 190 & 10 & - \\
\hline 4 & OI & 777.4 & 1.38 & 165 & 0.328 \\
\hline 5 & bg & 572.5 & 1.65 & 105 & 0.356 \\
\hline 6 & $\mathrm{Na}$ & 589.3 & 1.46 & 105 & 0.736 \\
\hline 7 & $\mathrm{H} \beta$ & 486.1 & 1.31 & 105 & 0.337 (at $486.1 \mathrm{~nm})$ \\
\hline \multicolumn{6}{|c|}{ Imager 9 (16 bit), Ithaca-campaign } \\
\hline 1 & OI & 557.7 & 1.71 & 50 & 0.356 \\
\hline 2 & OI & 630.0 & 1.65 & 120 & 0.405 \\
\hline 3 & $\mathrm{OH}$ & $>720$ & - & 1 & - \\
\hline 5 & bg & 572.5 & 1.77 & 50 & 0.343 \\
\hline \multicolumn{6}{|c|}{ Imager 10 (16 bit), Paratunka } \\
\hline 1 & OI & 557.7 & 1.73 & 105 & 0.285 \\
\hline 2 & OI & 630.0 & 1.58 & 165 & 0.402 \\
\hline 3 & $\mathrm{OH}$ & $>720$ & - & 10 & - \\
\hline 4 & OI & 777.4 & 1.40 & 165 & 0.227 \\
\hline 5 & bg & 572.5 & 1.74 & 105 & 0.297 \\
\hline 6 & $\mathrm{H} \beta$ & 486.1 & 1.32 & 105 & 0.305 (at $486.1 \mathrm{~nm})$ \\
\hline \multicolumn{6}{|c|}{ Imager 11 (16 bit), Magadan } \\
\hline 1 & OI & 557.7 & 1.68 & 105 & 0.327 \\
\hline 2 & OI & 630.0 & 1.64 & 165 & 0.480 \\
\hline 3 & $\mathrm{OH}$ & $>720$ & - & 10 & - \\
\hline 4 & OI & 777.4 & 1.42 & 165 & 0.290 \\
\hline 5 & bg & 572.5 & 1.77 & 105 & 0.355 \\
\hline 6 & $\mathrm{H} \beta$ & 486.1 & 1.33 & 105 & 0.348 (at $486.1 \mathrm{~nm})$ \\
\hline
\end{tabular}

Table 2. Stations of OMTIs. Geomagnetic values are calculated using the IGRF-10 mode at epoch 2005 at an altitude of $0 \mathrm{~km}$.

\begin{tabular}{|c|c|c|c|c|c|c|c|}
\hline Station & Abb. & $\begin{array}{c}\text { Geographic } \\
\text { latitude }\end{array}$ & $\begin{array}{c}\text { Geographic } \\
\text { longitude }\end{array}$ & $\begin{array}{c}\text { Magnetic } \\
\text { latitude }\end{array}$ & $\begin{array}{c}\text { Inclination } \\
\text { (degree) }\end{array}$ & $\begin{array}{c}\text { Declination } \\
\text { (degree) }\end{array}$ & $\begin{array}{c}\text { Observation } \\
\text { period }\end{array}$ \\
\hline Resolute Bay & RSB & 74.7 & 265.1 & 82.9 & 88.1 & -32.4 & Jan 2005- \\
\hline Athabasca & ATH & 54.7 & 246.7 & 61.7 & 76.6 & 17.4 & Sep 2005- \\
\hline Magadan & MGD & 60.0 & 150.9 & 51.9 & 71.5 & -11.1 & planned \\
\hline Paratunka & PTK & 52.9 & 158.3 & 45.7 & 64.5 & -6.5 & Aug 2007- \\
\hline Rikubetsu & RIK & 43.5 & 143.8 & 34.9 & 57.4 & -8.9 & Oct 1998- \\
\hline Shigaraki & SGK & 34.8 & 136.1 & 25.6 & 48.6 & -7.0 & Oct 1998- \\
\hline Sata & STA & 31.0 & 130.7 & 21.5 & 44.4 & -5.9 & Jul 2000- \\
\hline Yonaguni & YNG & 24.5 & 123.0 & 14.6 & 35.1 & -3.6 & Mar 2006- \\
\hline Kototabang & KTB & -0.20 & 100.3 & -10.4 & -19.8 & -0.3 & Oct 2002- \\
\hline Darwin & DRW & -12.4 & 131.0 & -21.8 & -39.9 & 3.5 & Oct 2001- \\
\hline
\end{tabular}

vations.

Figure 2 shows examples of the images of mesospheric gravity waves and medium-scale traveling ionospheric disturbances (MSTIDs) simultaneously observed at Shigaraki, Japan, on May 20, 2007. The gravity waves were seen in both images of $557.7 \mathrm{~nm}$ (altitude: $\sim 95 \mathrm{~km}$, left two panels) and in the $\mathrm{OH}$ band ( $\sim 86 \mathrm{~km}$, right two panels) throughout the night. They had a phase front of ESE-WNW and propagated northeastward. The horizontal wavelengths of the gravity waves were $\sim 20-30 \mathrm{~km}$. On the other hand, the MSTIDs seen in the 630.0-nm images (altitude: 200$300 \mathrm{~km}$, center two panels) had a much larger spatial scale of $\sim 100-400 \mathrm{~km}$, with some branching structures in the wave. They had a phase front of SSE-NNW and propa- gated southwestward. It is noteworthy that the two wave structures in the mesopause region and the thermosphere are completely different in scale and propagation direction.

\section{Propagation Directions of Waves}

From the sequential measurements of airglow images, such as those shown in Fig. 2, we can estimate spatial scales, propagation directions, and phase velocities of the waves in the mesopause region and the thermosphere. Such attempts have been made by using the OMTIs and other imagers in the Japanese longitudinal sector. Figure 3 summarizes the directions of the wave propagations obtained by airglow imagers in Japan, Indonesia, and Australia. All these results have been published in previous literatures, as 


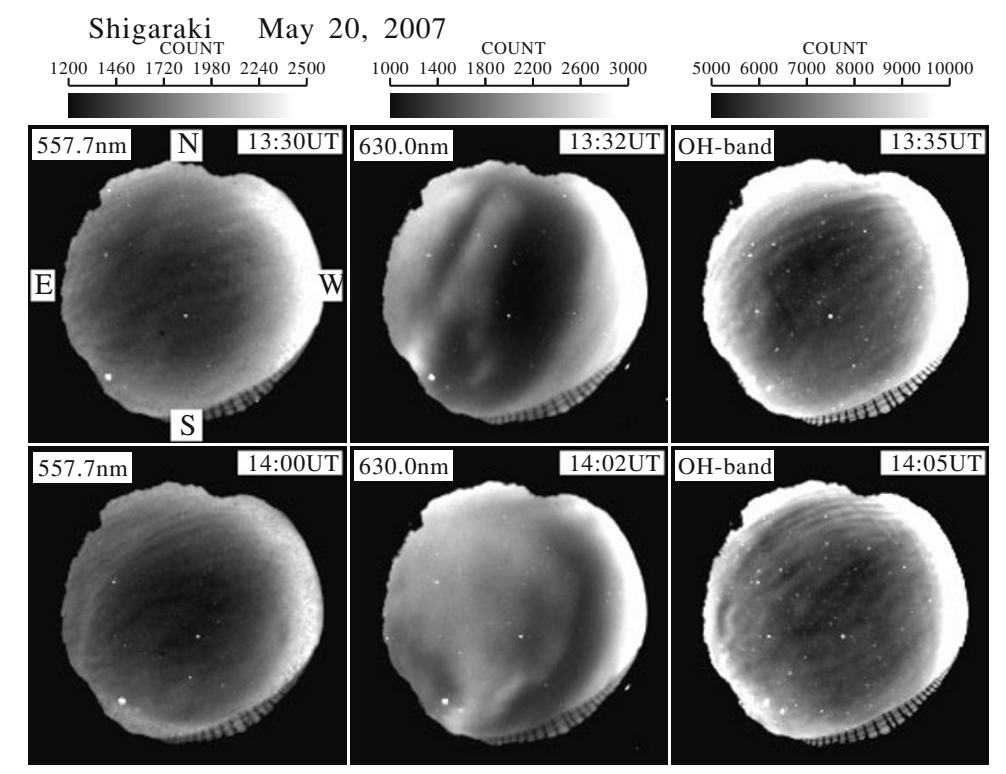

Fig. 2. All-sky airglow images obtained nearly simultaneously by imager 1 at Shigaraki, Japan, on May 20, 2007, for emissions of (left) $557.7 \mathrm{~nm}$, (center) $630.0 \mathrm{~nm}$, and (right) $\mathrm{OH}$ band. White dots are stars. Local time of Shigaraki is $9 \mathrm{~h}$ ahead of UT. For all images, upward is to the north and left is to the east. Small-scale gravity waves with ESE-WNW phase front are seen in the 557.7-nm and OH-band images, while intense southwestward-moving medium-scale traveling ionospheric disturbances are seen in the 630.0-nm images.

Table 3. References of wave observations shown in Fig. 3.

\begin{tabular}{ccc}
\hline Station & Latitude & Reference \\
\hline \hline & Mesosphere $(557.7 \mathrm{~nm}$ and OH-band) \\
\hline Rikubetsu, Japan & $43.5^{\circ} \mathrm{N}$ & Ejiri et al. (2003) \\
Shigaraki, Japan & $34.8^{\circ} \mathrm{N}$ & Nakamura et al. $(1999) ;$ Ejiri et al. (2003) \\
Kototabang, Indonesia & $0.2^{\circ} \mathrm{S}$ & Suzuki (2007) \\
Tanjungsari, Indonesia & $6.9^{\circ} \mathrm{S}$ & Nakamura et al. (2003) \\
Darwin, Australia & $12.4^{\circ} \mathrm{S}$ & Suzuki et al. (2004) \\
Adelaide, Australia & $24^{\circ} \mathrm{S}$ & Walterscheid et al. (1999) \\
\hline & Thermosphere (630.0 nm) \\
\hline Rikubetsu, Japan & $43.5^{\circ} \mathrm{N}$ & Shiokawa et al. (2003a) \\
Shigaraki, Japan & $34.8^{\circ} \mathrm{N}$ & Shiokawa et al. (2003a) \\
Sata, Japan & $31.0^{\circ} \mathrm{N}$ & Otsuka et al. (2004); Shiokawa et al. (2005) \\
Okinawa, Japan & $26.9^{\circ} \mathrm{N}$ & Shiokawa et al. (2002) \\
Yonaguni, Japan & $24.5^{\circ} \mathrm{N}$ & Ogawa et al. (2008) \\
Kototabang, Indonesia & $0.2^{\circ} \mathrm{S}$ & Shiokawa et al. (2006) \\
Darwin, Australia & $12.4^{\circ} \mathrm{S}$ & Otsuka et al. (2004); Shiokawa et al. (2005) \\
Renner Springs, Australia & $18.3^{\circ} \mathrm{S}$ & Shiokawa et al. (2005) \\
\hline
\end{tabular}

cited in the following sections and in Table 3. Compiling these different results on a global map gives additional insights into the generation and propagation of the gravity waves in the mesosphere and the thermosphere.

\subsection{Gravity waves in the nighttime mesopause region}

Figure 3(a) shows the propagation characteristics of the gravity waves in the mesopause region. In Japan, Nakamura et al. (1999) were the first to report gravity wave motions from 18-month imaging observation of the $\mathrm{OH}$ airglow at Shigaraki $\left(34.8^{\circ} \mathrm{N}\right)$. Their imager did not belong to the OMTIs and had a narrower field of view of $93.9^{\circ} \times 93.9^{\circ}$ in north-south and east-west directions. They separated the observed waves with wavelengths smaller and larger than $17.5 \mathrm{~km}$. The former are ripples, most likely caused by local instabilities. The latter are probably gravity waves.
For the latter waves with larger horizontal wavelengths of $\sim 17.5-60 \mathrm{~km}$, the horizontal propagation direction showed clear seasonal variation with eastward to northward in summer and all westward (NW, W, and SW) in winter. These waves had short periods $(5-30 \mathrm{~min})$ with horizontal phase speeds of $0-100 \mathrm{~m} / \mathrm{s}$.

Ejiri et al. (2003) extended the study by Nakamura et al. (1999), using the two all-sky imagers (imagers 1 and 3) of the OMTIs at Shigaraki and Rikubetsu $\left(43.5^{\circ} \mathrm{N}\right)$, Japan, for both 557.7-nm and OH-band airglow images obtained over one year in 1998-1999. They studied gravity waves with horizontal wavelengths less than $\sim 40 \mathrm{~km}$ by subtracting spatial running averages of $40 \times 40 \mathrm{~km}$ from raw airglow images. The propagation directions of gravity waves in the summer were either northward or northeastward at 

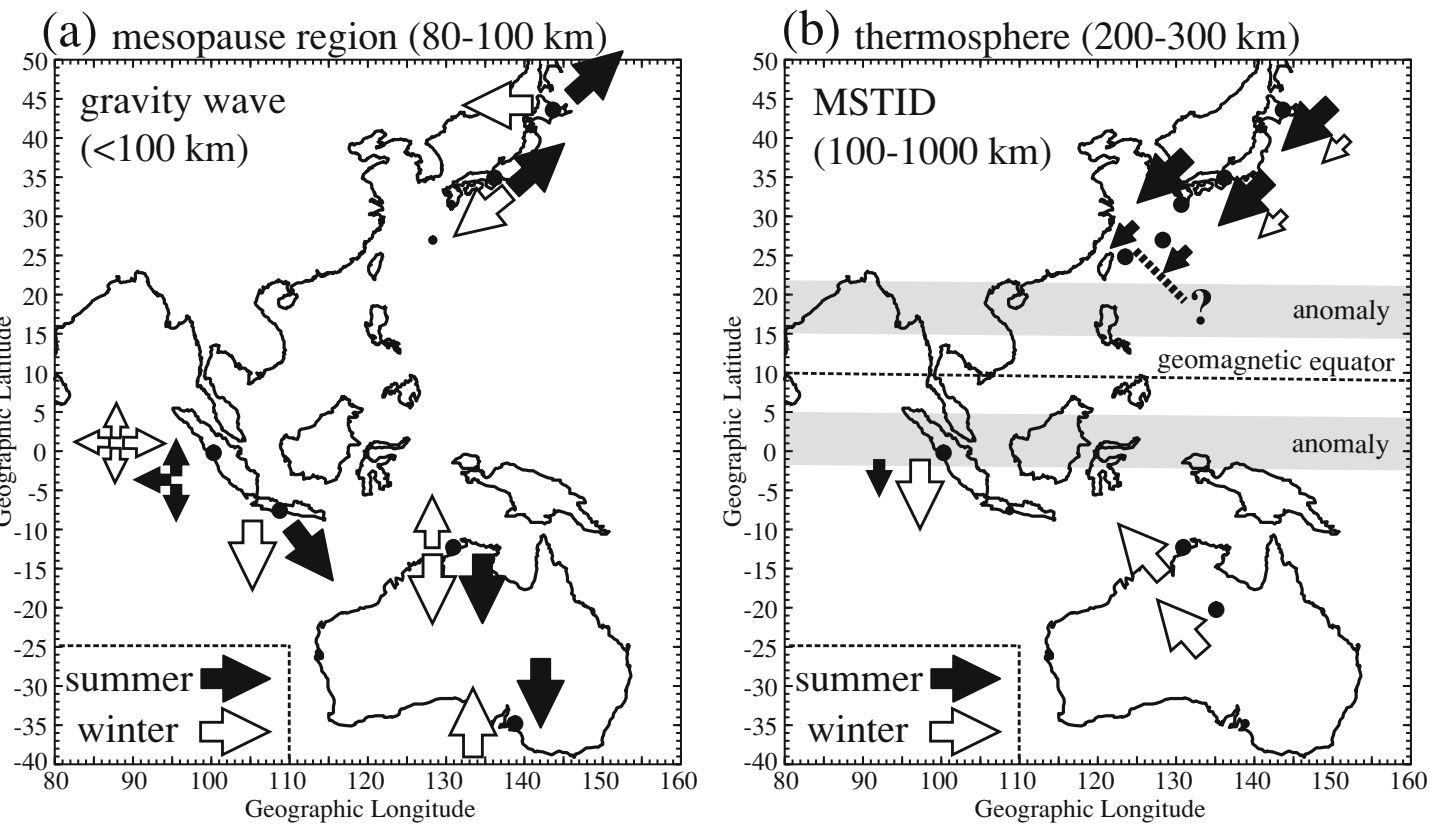

Fig. 3. Schematic picture of the direction of wave propagations observed by airglow images in summer and winter of each hemisphere, (a) for gravity waves with horizontal scales less than $100 \mathrm{~km}$ in the mesopause region (in 557.7-nm and OH-band airglow images) and (b) for MSTIDs with horizontal scales of $100-1,000 \mathrm{~km}$ in the thermosphere (in 630.0-nm airglow images). The size of the arrows schematically indicates the relative occurrence of waves for each direction.

both locations. However, in winter, the propagation directions at Rikubetsu were generally westward (NW, W, and $\mathrm{SW}$ ), whereas those at Shigaraki were only southwestward. These waves had dominant phase speeds of $\sim 20-50 \mathrm{~m} / \mathrm{s}$. These directions of wave propagations are shown by arrows over Japan in Fig. 3(a). Shiokawa et al. (2000b) reported simultaneous imaging events of mesospheric gravity waves at three stations in Japan (Moshiri, Shigaraki, and Bisei) and concluded that similar gravity-wave characteristics were observed at these stations, when background wind patterns in the mesosphere, observed at the northern and southern edges of Japan, were similar.

In the Australian continent, two reports have been published regarding gravity wave propagation in the mesopause region, as shown in Fig. 3(a). Walterscheid et al. (1999) reported 10-month observations of $\mathrm{OH}$ and $\mathrm{O}_{2}$ airglow images at Adelaide, Australia $\left(24^{\circ} \mathrm{S}, 138^{\circ} \mathrm{E}\right)$. The emission layer of the $\mathrm{O}_{2}$ atmospheric band at $\sim 866 \mathrm{~nm}$ is $\sim 94 \mathrm{~km}$ in the mesopause region. Walterscheid et al. (1999) showed that during the Australian summer, small-scale waves in the airglow images were predominantly poleward-propagating, while during the Australian winter they were predominantly equatorward. The horizontal wavelengths were typically a few tens of kilometers, while the measured phase speeds were typically $\sim 50-80 \mathrm{~m} / \mathrm{s}$. Observed periods of $\sim 5-$ 20 min were typical.

Suzuki et al. (2004) studied the propagation direction of gravity waves observed by imager 4 of the OMTIs at Darwin $\left(12.4^{\circ} \mathrm{S}\right)$, Australia, for both 557.7-nm and $\mathrm{OH}-$ band airglow images obtained over 11 months in 2001-2002. They showed that most of the observed waves propagated in the meridional direction, poleward (southward) in the Australian summer and both poleward and equatorward in the Australian winter. The waves they analyzed had wavelengths of less than $90 \mathrm{~km}$ (peak: $30-50 \mathrm{~km}$ ) and phase velocities of less than $90 \mathrm{~m} / \mathrm{s}$ (peak: $30-60 \mathrm{~m} / \mathrm{s}$ ).

At equatorial latitudes, two results were obtained based on airglow imaging observations in Indonesia, as shown in Fig. 3(a). Nakamura et al. (2003) studied gravity wave propagations observed in $\mathrm{OH}$ airglow images at Tanjungsari $\left(6.9^{\circ} \mathrm{S}, 107.9^{\circ} \mathrm{E}\right)$, West Jawa, Indonesia, for one year in 2000-2001. They used the same $\mathrm{OH}$ imager as that used at Shigaraki by Nakamura et al. (1999). Propagation directions of the observed gravity waves were mostly southward, although there were some exceptions of eastwardpropagating waves between December and February. The period, horizontal wavelength, and observed horizontal phase speeds of the observed gravity waves were typically 5-13 $\mathrm{min}, 13-45 \mathrm{~km}$, and 37-75 m/s, respectively.

Suzuki (2007) studied gravity wave propagation using airglow images of both $557.7 \mathrm{~nm}$ and $\mathrm{OH}$ band obtained by imager 5 of OMTIs at Kototabang $\left(0.2^{\circ} \mathrm{S}\right)$, West Sumatra, Indonesia, for two years in 2003-2005. Due to cloudy conditions at the station, only 26 nights of data were available. The observed gravity waves propagated in almost all directions in the southern-hemispheric winter (May-August), with a slight preference for the eastward direction, while they propagated in all directions except eastward in the southern-hemispheric summer (November-February). The horizontal wavelength and phase speed of the waves were $40-80 \mathrm{~km}$ and $40-80 \mathrm{~m} / \mathrm{s}$, respectively.

Figure 3(a) is the summary of these previous observations of gravity wave propagation directions in Japan, Australia, and Indonesia. As discussed by Nakamura et al. (1999) and Ejiri et al. (2003), the preference of eastward in the summer and westward in the winter over Japan indicates 
that these waves are propagated from the lower atmosphere and filtered in the middle atmosphere by the mean winds (mesospheric jet). During the Japanese winter, low pressure cell tends to exist in the northeast of Japan. Thus, the southwestward preference of gravity waves in winter over Japan may be also explained by this pressure distribution in the troposphere. The slight difference in meridional direction in winter between Shigaraki (southwestward) and Rikubetsu (toward NW, W, and SW) may indicate that the latitude of Rikubetsu is closer to the source region of these waves.

Similar zonal tendency (eastward in summer and westward in winter) was also weakly seen in statistical results by Walterscheid et al. (1999) at Adelaide, although the preference was mainly in the meridional direction. As discussed by Walterscheid et al. (1999) and Suzuki et al. (2004), the poleward- (southward-) moving waves over the Australian continent in summer are likely to be generated by strong convective activities in the equatorial latitudes, propagating in the mesospheric thermal duct over long distance. The results in Indonesia also indicate the convective source in the equatorial zone, causing the poleward preference of waves for both summer and winter at Tanjungsari and less preference of wave directions at Kototabang, which is located in the convective source region. The relative location from the tropospheric convective source identified by satellite cloud images to these Indonesian stations was consistent with the observed wave propagation directions, as shown by Nakamura et al. (2003) and Suzuki (2007). The observations in Japan also suggest the poleward propagation of the waves in the summer, similar to the observations in Australia. These results of propagation directions of small-scale waves with wavelengths less than $100 \mathrm{~km}$ indicate that small-scale gravity waves in the mesopause region are strongly controlled by wind filtering, ducting, and relative location from the tropospheric wave source. There are still some questions regarding these propagation maps, i.e., (1) why do the waves propagate mainly in the meridional direction in Australia and the zonal direction in Japan, (2) what is the source of the equatorward waves in winter at Darwin, Adelaide, and Shigaraki? These may be because of the relative location to the local wave sources, such as high mountains and tropospheric/mesospheric jets.

\subsection{Waves in the nighttime thermosphere}

Figure 3(b) summarizes the propagation directions of waves in the thermosphere observed in the 630.0-nm airglow images at various locations. At midlatitudes in Japan and Australia, the major wave structures seen in the 630.0nm images are nighttime MSTIDs that have a NW-SE phase front and that propagate southwestward, as shown by the example in Fig. 2. Shiokawa et al. (2003a) made a statistical study of the MSTIDs in the 630.0-nm airglow images obtained by imagers 1 and 3 at Shigaraki and Rikubetsu in 1998-2000. They found that most of the observed MSTIDs in the $630.0-\mathrm{nm}$ images propagated southwestward. The typical wavelength, velocity, period, and amplitude were $100-300 \mathrm{~km}, 50-100 \mathrm{~m} / \mathrm{s}, 0.5-1.5 \mathrm{~h}$, and 5-15\%, respectively. The occurrence rate had a major peak (50-60\%) in the summer that appeared $\sim 2$ months earlier at lower latitudes and a minor peak in winter.
Kubota et al. (2000) and Saito et al. (2001) concluded that the MSTIDs can propagate more than 1,000 km over Japan, on the basis of large-scale MSTID images over Japanese islands obtained by multipoint airglow imaging observations and network GPS receivers during the $F$ region Radio and Optical measurement of Nighttime TIDs (FRONT) campaign. Shiokawa et al. (2002) made an airglow imaging observation using imager 2 of OMTIs at Okinawa $\left(26.9^{\circ} \mathrm{N}, 128.3^{\circ} \mathrm{E}\right)$ during the FRONT-2 campaign of August 4-15, 1999. They showed two events for which the MSTIDs reached and did not reach Okinawa from higher latitudes. For the latter case when the MSTIDs did not reach Okinawa, an intense 630.0-nm airglow band, which corresponds to the equatorial ionospheric anomaly crest, was observed in the southern sky of Okinawa. Thus, it seems that the MSTIDs propagate southwestward at midlatitudes, and the equatorial anomaly is a possible southern boundary of the midlatitude MSTIDs. Ogawa et al. (2008) reported an MSTID event observed by imager 8 of OMTIs at Yonaguni $\left(24.5^{\circ} \mathrm{N}\right)$, during which the anomaly was not in the field of view of the imager.

These MSTIDs at midlatitudes are accompanied by an oscillating electric field, as shown by Shiokawa et al. (2003b) using simultaneous data from imager 1 of OMTIs at Shigaraki and from the ion drift meter on board the DMSP satellite that passed in the field of view of imager 1. Otsuka et al. (2004) were the first to show that this electric field was mapped to the other hemisphere along the geomagnetic field line and formed a conjugate MSTID structure in the Australian continent, by using simultaneous imaging measurements of MSTIDs at Sata $\left(31.0^{\circ}\right)$, Japan (imager 2), and Darwin, Australia (imager 4). Shiokawa et al. (2005) further confirmed this clear conjugacy for all MSTIDs observed during the FRONT-3 campaign period ( $\sim 1$ month) using two pairs of geomagnetically conjugate stations at Sata (imager 2)-Darwin (imager 4) and Shigaraki (imager 1)-Renner Springs (imager 6, $18.3^{\circ} \mathrm{S}$, $133.8^{\circ}$ ). Thus, the statistical characteristics of MSTIDs in Japan reported by Shiokawa et al. (2003a) are likely to be mapped to the southern hemisphere in Australia. Actually, Kotake et al. (2006) have shown, using total electron content (TEC) data obtained by multipoint GPS receivers, that in the Japanese and Australian longitudinal sector, nighttime MSTIDs are most active near the June solstice (summer in Japan and winter in Australia). These propagation and occurrence characteristics of MSTIDs are indicated by arrows in Fig. 3(b).

At equatorial latitudes, Shiokawa et al. (2006) newly found quasi-periodic southward-moving waves in the $630.0-\mathrm{nm}$ airglow images obtained by imager 5 at Kototabang, Indonesia, based on 2 years of observations in 2002-2004. The waves have predominantly east-west phase fronts and repeatedly propagate southward with a velocity of $310 \pm 110 \mathrm{~m} / \mathrm{s}$ and a period of $40 \pm 15 \mathrm{~min}$, giving a horizontal scale size of $\sim 700 \mathrm{~km}$. They are frequently observed in May-July (winter in the southern hemisphere), with an occurrence rate of 53\%, and are also observed in other seasons with occurrences of $\sim 20 \%$.

Figure 3(b) summarizes the above observations of the MSTIDs and waves in the 630.0-nm airglow images at alti- 
tudes of 200-300 km. The waves observed at midlatitudes tend to propagate southwestward in the northern hemisphere and northwestward in the southern hemisphere during the nighttime. These midlatitude wave structures have geomagnetic conjugacy, most prominent in the northern hemispheric summer. At Kototabang, Indonesia, which is in the equatorial anomaly region, southward-moving waves were frequently observed in, and poleward of, the anomaly crest. It is not clear how these two types of waves are coupled poleward of the anomaly. Other than these waves, distinct airglow depletions caused by equatorial plasma bubbles are often observed at Kototabang, Sata, Darwin, and even Shigaraki (e.g., Otsuka et al., 2002, 2006; Shiokawa et al., 2004; Ogawa et al., 2005).

Since the 630.0-nm airglow intensity varies according to the height variations of the $F$ region ionosphere, the observed waves may be oscillations of the ionized atmosphere rather than the neutrals. For midlatitude MSTIDs, one of the plausible mechanisms that can cause the NW-SE phase front of the waves is the Perkins instability, which is basically ionospheric plasma instability due to inhomogeneities of the ionospheric conductivity and electric current (e.g., Perkins, 1973; Miller et al., 1997). However, the linear growth rate of the Perkins instability is very small (e.g., Shiokawa et al., 2003b). Moreover, this instability does not explain the observed scale size of the MSTIDs and preference of southwestward motion. Since the Perkins instability is a sort of Rayleigh-Taylor type instability, the instability structure seeded by gravity waves can move with the motion of the seed gravity waves. Filtering of the gravity waves from the lower atmosphere by the nighttime thermospheric diurnal tide may cause westward preference of the wave directions. The instability structure can also move according to the background electric field, as suggested by Kelley and Makela (2001), irrespective to the seed gravity wave motion. Seeding of the instability by gravity waves from the lower atmosphere and electrodynamical coupling of ionospheric $F$ and $E$ regions in both hemispheres would be important for solving this problem.

For the waves in the equatorial region, it is noteworthy that northward-propagating waves were rarely observed at Kototabang. Shiokawa et al. (2006) suggested that gravity waves in the lower thermosphere at altitudes below $\sim 300 \mathrm{~km}$ are a plausible cause of the observed southwardmoving waves, because of the observed east-west phase front and the westward tilt of the front for some cases. However, some explanation is needed for the systematic southward motion. This southward motion is different from the propagation directions of gravity waves in the mesopause region shown in Fig. 3(a).

The overall propagation characteristics of waves in the mesopause region and the thermosphere are quite dissimilar. It should be noted that the scale size of the analyzed waves $(<100 \mathrm{~km}$ in the mesopause region and 100 $1,000 \mathrm{~km}$ in the thermosphere) are different. The vertical thicknesses of the 630.0-nm and 557.7-nm airglow layers are $\sim 100 \mathrm{~km}$ and $\sim 10 \mathrm{~km}$, respectively. It would be difficult to observe small-scale waves with vertical wavelengths less than $100 \mathrm{~km}$ in the 630.0-nm airglow images due to the effect of line-of-sight integration. This thicker layer width of 630.0-nm emission may make it difficult to observe small-scale gravity waves in the thermosphere. In addition, dissipative gravity wave theory shows that gravity waves with $\lambda_{\mathrm{z}}<100 \mathrm{~km}$ dissipate fairly rapidly in the $F$-region (Vadas, 2007).

On the other hand, it may be possible to identify waves with scale sizes larger than $100 \mathrm{~km}$ in the mesospheric airglow images. Such larger-scale waves in the mesosphere have not been studied using airglow images. One should be careful in analyzing the larger scale waves in the mesosphere, because the scale size of the wave becomes comparable to the various inhomogeneities inherent in all-sky images, such as the van Rhijn effect, atmospheric extinction, and inhomogeneities in sensitivity caused mainly by the fish-eye lens. All these inhomogeneities vary from the center to the edge of the images and have "wavelengths" comparable to that of the mesospheric gravity waves with wavelengths larger than $\sim 100 \mathrm{~km}$. By subtracting running averages of sequential images, however, one can identify larger-scale waves in the mesospheric airglow images. In the next section, we show an example of such an analysis to investigate the penetration of large-scale waves from the mesosphere to the thermosphere.

\section{A Comparison of Mesospheric and Thermo- spheric Waves at the Equator}

The southwestward-moving nighttime MSTIDs at midlatitudes are more likely to be caused by ionospheric instabilities rather than gravity waves in the thermosphere (e.g., Fukao et al., 1991; Kelley and Fukao, 1991; Miller et al., 1997; Shiokawa et al., 2003b). It may be difficult to identify the gravity waves that cause seeding of the instabilities in the thermosphere and the mesosphere, because the observed MSTIDs motions may not reflect the motion of the seed gravity waves. On the other hand, the quasiperiodic southward-moving waves studied by Shiokawa at al. (2006) in the 630.0-nm airglow images at Kototabang, Indonesia, seem to be directly caused by gravity waves in the thermosphere. Thus, it would be worthwhile to compare the airglow images of $557.7 \mathrm{~nm}$ and $\mathrm{OH}$ band in the mesosphere and $630.0 \mathrm{~nm}$ in the thermosphere during the southward-moving wave event observed at Kototabang.

\subsection{Observations}

Figure 4 shows such a comparison for the quasi-periodic southward-moving waves observed at Kototabang, Indonesia, on May 16, 2004. This event was studied in detail by Shiokawa et al. (2006) in their Figs. 2 and 3. In Fig. 4, we compare the north-south cross sections (keograms) of allsky images of (a) OH-band, (b) $630.0 \mathrm{~nm}$, and (c) $557.7 \mathrm{~nm}$ during this night, by subtracting 1-h running averages from the raw images. This subtraction was made by calculating $\left(I(t)-I_{a(t)}\right) / I_{a(t)}$, where $I(t)$ and $I_{a(t)}$ are airglow intensity at time $t$ and average intensity over $t \pm 30 \mathrm{~min}$, respectively. As discussed in the previous section, this method of 1-h running-average subtraction makes it possible to focus on the waves with periods less than $1 \mathrm{~h}$ and to correct the spatial inhomogeneities of the images.

The quasi-periodic southward-moving waves are clearly seen in Fig. 4(b) in the 630.0-nm keograms throughout the night and are most intensified at 1800-1900 UT. A similar 


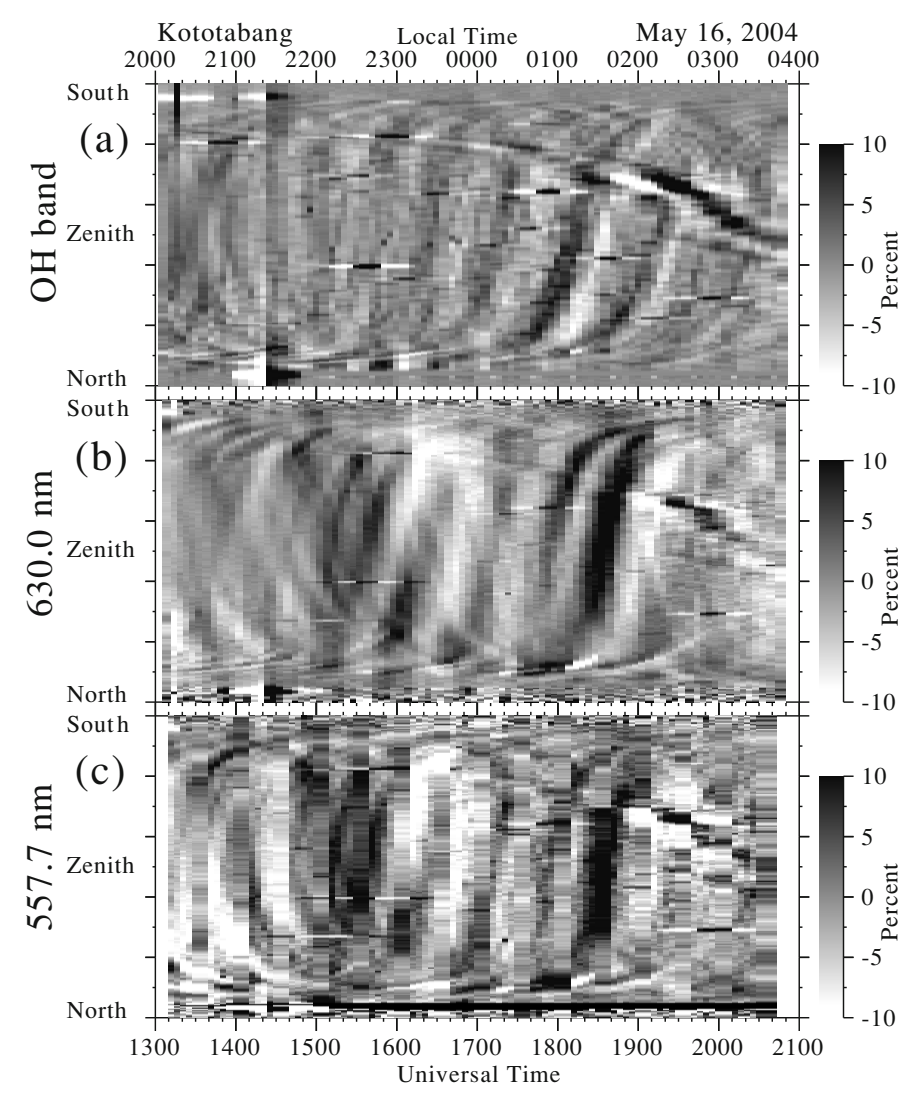

Fig. 4. North-south cross sections (keograms) of airglow images in the raw all-sky coordinates for emissions of (a) OH band, (b) $630.0 \mathrm{~nm}$, and (c) $557.7 \mathrm{~nm}$, obtained by imager 5 at Kototabang, Indonesia, on May 16, 2004. The keograms indicate deviations from 1-h running averages in percent. Southward-moving waves are seen in all three emissions, particularly after 1700 universal time (UT). The structure gradually moving northward at just north of zenith at 1700-2100 UT is the Milky Way.

structure can be seen in the 557.7-nm keogram in Fig. 4(c). However, it should be noted that the wave-phase peaks at these two line emissions are at exactly the same timing at the same location in this all-sky coordinate. This fact indicates that the structures seen in the 557.7-nm emission are not from the mesopause region but are due to the contamination from the thermospheric 557.7-nm emission. In other words, the imager observed the same gravity waves in the thermosphere through 630.0-nm and 557.7-nm emissions. At equatorial latitudes particularly near the anomaly region, there are significant amounts of 557.7-nm emission from the thermosphere at altitudes of $200-300 \mathrm{~km}$ (e.g., Shepherd et al., 1997). The major source of the 630.0-nm emission, i.e., dissociative recombination of $\mathrm{O}_{2}^{+}$ $\left(\mathrm{O}_{2}^{+}+\mathrm{e} \rightarrow 2 \mathrm{O}^{*}\right)$, causes both the excited states of $\mathrm{O}\left({ }^{1} \mathrm{D}\right)$ (source of $630.0 \mathrm{~nm}$ ) and $\mathrm{O}\left({ }^{1} \mathrm{~S}\right)$ (source of $\left.557.7 \mathrm{~nm}\right)$. This contamination of the thermospheric green line makes it difficult to study the penetration of mesospheric gravity waves into the thermosphere using the 557.7-nm airglow images.

Figure 4(a) shows that the quasi-periodic southward waves can be also seen in the near-infrared $\mathrm{OH}$ band keograms. Since the broadband filter to obtain OH-band images of imager 5 passes only the wavelengths at 720$910 \mathrm{~nm}$, as shown in Table 2, this structure cannot be due to the contamination of the thermospheric 557.7-nm and 630.0-nm emissions. Moreover, the phase peaks of the southward-moving waves in the $\mathrm{OH}$-band keogram are at different locations from those in the 630.0-nm keogram in the all-sky coordinates. From these considerations, we conclude that the southward-moving waves seen in the $\mathrm{OH}$ band keogram are the gravity waves in the mesopause region at altitudes of $\sim 86 \mathrm{~km}$.

To see the wave characteristics in Fig. 4 more clearly, we made keograms in geographical coordinates in Fig. 5 for both $\mathrm{OH}$-band and 630.0-nm airglow emissions for the intensity (Figs. 5(b) and 5(d) in count and Rayleigh) and deviations from 1-h running averages (Figs. 5(a) and 5(c)). The vertical axis is in geographic latitudes of $5^{\circ}$ centered at Kototabang. Similar keograms with a wider vertical axis $\left(12^{\circ}\right)$ are shown in Fig. 2 of Shiokawa et al. (2006) for the 630.0-nm emission.

The enhancement of emission intensity at 1400-1600 UT in the 630.0-nm keogram in Fig. 5(d) is the equatorial anomaly crest, moving toward the geomagnetic equator, which is $\sim 10^{\circ} \mathrm{N}$ in geographic latitude. As pointed out by Shiokawa et al. (2006), the southward-moving waves with a period of $\sim 40 \mathrm{~min}$ are seen in the 630.0-nm keogram throughout the night in Fig. 5(c). The waves are most clear at $\sim 1600$ UT and 1800-2000 UT. Some waves can be recognized in the raw intensity data in Fig. 5(d). The horizontal phase velocity of the southward motion is $\sim 300 \mathrm{~m} / \mathrm{s}$.

In Fig. 5(a), the southward-moving waves with a slightly longer period of $\sim 50 \mathrm{~min}$ are seen after $1600 \mathrm{UT}$ (2300 LT) in the OH-band keogram. The waves are most clear at 1700-1900 UT (0000-0200 LT). These waves can be recognized in the raw count keogram in Fig. 5(b), although 


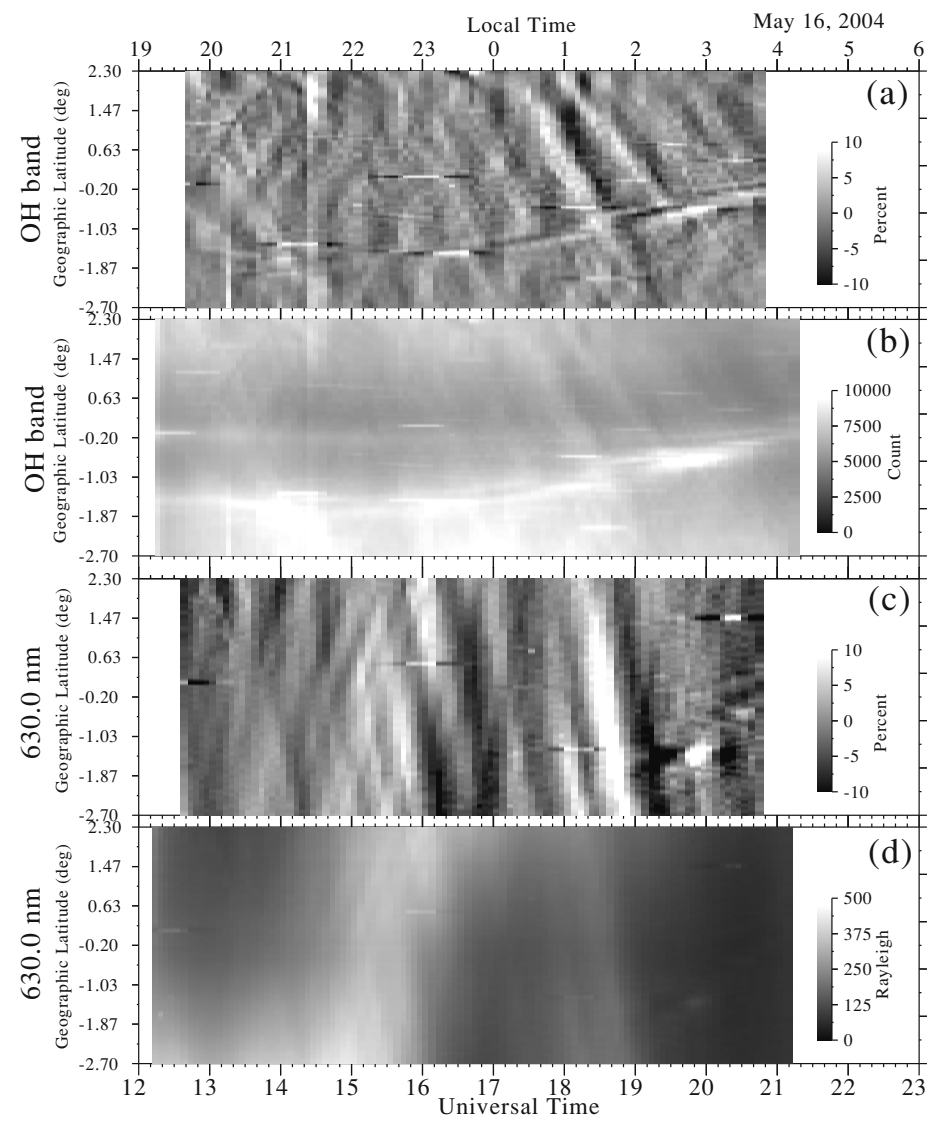

Fig. 5. North-south cross sections (keograms) of airglow images in the geographical coordinates at latitudes from $2.7^{\circ} \mathrm{S}$ to $2.3^{\circ} \mathrm{N}$ for emissions of $\mathrm{OH}$ band and $630.0 \mathrm{~nm}$ obtained by imager 5 at Kototabang, Indonesia, on May 16, 2004. From top to bottom, (a) deviations from 1-h running averages for the $\mathrm{OH}$ band, (b) raw counts for the $\mathrm{OH}$ band, (c) deviations from 1-h running averages for $630.0 \mathrm{~nm}$, (d) raw intensities (Rayleigh) for 630.0 nm. Bright emissions in the bottom half of panel (b) are the Milky Way.

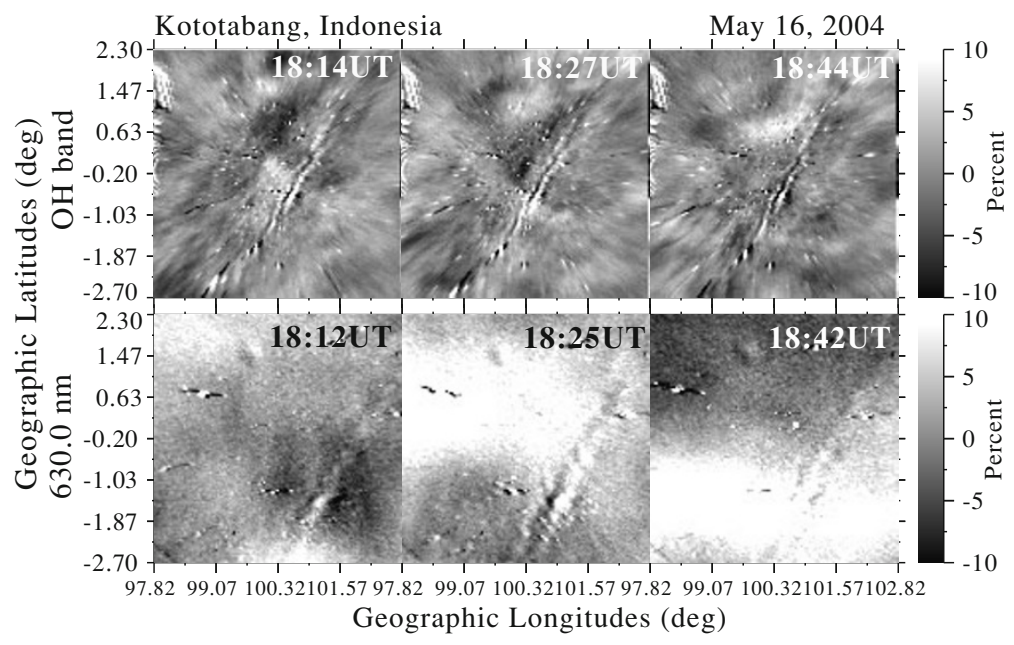

Fig. 6. Airglow images in geographical coordinates for the $\mathrm{OH}$ band in the top three panels and for $630.0 \mathrm{~nm}$ in the bottom three panels, obtained nearly simultaneously at Kototabang, Indonesia, on May 16, 2004. These images are deviations from 1-h running averages. The narrow-band structures from southeast (bottom left) to northwest (top right) in all images are the Milky Way.

the bottom half of Fig. 5(b) is significantly contaminated by intense emission from the galaxy. The phase velocity of the waves at $1700-1900 \mathrm{UT}$ is $50-70 \mathrm{~m} / \mathrm{s}$. The period of $\sim 50 \mathrm{~min}$ and phase velocity of $50-70 \mathrm{~m} / \mathrm{s}$ give horizontal wavelengths of $150-210 \mathrm{~km}$.

Figure 6 shows images of the $\mathrm{OH}$ band and $630.0 \mathrm{~nm}$ in geographical latitudes and longitudes $\left(5^{\circ} \times 5^{\circ}\right)$ for the southward-moving waves at 1800-1900 UT in the keograms of Figs. 5(a) and 5(c). One-hour running averages were subtracted. The southward-moving wave is very clear in the $630.0-\mathrm{nm}$ images in the bottom three panels. The wavelength $(\sim 700 \mathrm{~km})$ of the wave is larger than the plotted area. Images with a wider area $\left(12^{\circ} \times 12^{\circ}\right)$ are shown in Fig. 3 of Shiokawa et al. (2006). The amplitude of the wave 
in the 630.0-nm images was 10-20\%.

On the other hand, the wave structures in the OH-band images are less clear. The two phase peaks of southwardmoving waves in the keogram of Fig. 5(a) at 1800-1900 UT are seen near the center longitudes of $100^{\circ} \mathrm{E}$ at $1.5^{\circ} \mathrm{N}$ and $0.2^{\circ} \mathrm{S}$ in the image at $1814 \mathrm{UT}$. The amplitude of these waves in the $\mathrm{OH}$-band images was less than $10 \%$.

\subsection{Discussion}

Quasi-periodic southward-moving waves were observed simultaneously in the mesopause region ( $\mathrm{OH}$ band) and in the thermosphere $(630.0 \mathrm{~nm})$ on May 16, 2004, at Kototabang, Indonesia. The period, phase velocity, and wavelengths of the waves were $\sim 40 \mathrm{~min}, \sim 300 \mathrm{~m} / \mathrm{s}$, and $\sim 700 \mathrm{~km}$, respectively, in the thermosphere, while they were $\sim 50 \mathrm{~min}, 50-70 \mathrm{~m} / \mathrm{s}$, and $150-210 \mathrm{~km}$, respectively, in the mesopause region. These phase velocities and horizontal wavelengths are quite different. As estimated by Shiokawa et al. (2006), the observed wave in the thermosphere with the fast phase speed of $\sim 300 \mathrm{~m} / \mathrm{s}$ tends to become evanescent at altitudes below $100 \mathrm{~km}$. Thus, it is not likely that the observed waves with horizontal wavelengths of $150-210 \mathrm{~km}$ in the mesopause region directly penetrate into the thermosphere to cause the observed $\sim 700-\mathrm{km}$ scale waves in the 630.0-nm images. However, the 700$\mathrm{km}$ thermospheric gravity waves may be excited from thermospheric body forces, which are created from the dissipation of the smaller-scale 150-210 km waves which propagate from the mesopause and dissipate near the bottomside of the $F$-region, at altitudes of 180-200 km (Vadas and Fritts, 2006). Fritts et al. (2002) and Vadas et al. (2003) discussed secondary wave generation near the mesopause region driven by body forcing in association with the breaking of small-scale gravity waves from the lower atmosphere. The southward-moving waves observed in the $\mathrm{OH}$ images would cause southward body forcing when they break and may cause a secondary wave with southward phase velocity. Since a background wind profile was not available for this event, it is difficult to discuss further the propagation and breaking of the observed wave in the mesopause region.

It should be noted that the amplitude of the secondary wave is usually very small, only an order of percent of the original wave. However, upward propagation of the secondary wave from $100 \mathrm{~km}$ (mesopause region) to $250 \mathrm{~km}$ (630.0 nm airglow layer) would cause significant enhancement of the wave amplitudes because of decreasing neutral density. Assuming an average scale height of $30 \mathrm{~km}$ at this altitude range, the amplitude becomes $\exp ((250-100) /(2 \times$ $30))=12$ times larger from $100 \mathrm{~km}$ to $250 \mathrm{~km}$.

It should be also noted that a small-scale gravity wave typically takes $0.5-1.5$ hours to propagate from the mesopause region to the thermosphere. During the propagation, the wave can propagate several hundreds of kilometers horizontally, which can be out of field-of-view of the all-sky imagers. We may need to take these time and location effects into account when we investigate penetration of gravity waves by comparing airglow images obtained simultaneously in the mesosphere and the thermosphere at a station. Usually when we observe gravity waves in the airglow images, however, the waves cover most of the sky in the field of view and last for more than hours, as shown in the present case in Fig. 5.

\section{Summary and Discussion}

In this paper, we reviewed nighttime airglow imaging observations done since 1997 by OMTIs and other imagers in the Japanese longitudinal sector. Except for some degradations of sensitivity, the all-sky cooled-CCD imagers of OMTIs are working fairly well at various stations in Japan, Indonesia, Australia, and Canada. The latest information on the stations and quick-look plots of the data obtained by OMTIs are available at http://stdb2.stelab.nagoyau.ac.jp/omti/index.html.

Gravity waves in the middle atmosphere are dissipated near the mesopause region and cause acceleration/deceleration of mean wind (e.g., Vincent, 1984; Tsuda et al., 1990). Suzuki et al. (2007) estimated the horizontal momentum flux carried by the gravity waves in $\mathrm{OH}$ airglow images obtained at Shigaraki on November 19, 1999 by imager 1 . They estimated the momentum flux on this night to be $1-15 \mathrm{~m}^{2} / \mathrm{s}^{2}$ with an average of $4.9 \mathrm{~m}^{2} / \mathrm{s}^{2}(\sim 1.7 \mathrm{~m} / \mathrm{s} / \mathrm{h})$ and concluded that small-scale gravity waves seen in the airglow images can carry a significant amount of momentum flux to the mesopause region. The observed propagation directions at various locations in Fig. 3(a) indicate the directions of mean wind acceleration by the observed gravity waves.

The seasonal variation of zonal propagation directions of gravity waves over Japan indicates that strong wind filtering occurs in the middle atmosphere due to the mesospheric jet. This implies that, in the mesopause region, the mean wind is accelerated in directions opposite the mesospheric jet. This result is consistent with those for longer-period waves observed by the MU radar at Shigaraki (Tsuda et al., 1990). On the other hand, if the poleward-moving waves observed at Darwin and Adelaide in summer are ducted, as suggested by Walterscheid et al. (1999) and Suzuki et al. (2004), they would not carry momentum flux to the mesopause region. The features of propagation directions in Fig. 3(a) also suggest that gravity waves are generated in the equatorial convective zone and propagate poleward at both hemispheres. Such waves may cause acceleration of mean wind in the meridional direction (poleward) in the mesopause region. The Coriolis force would change this meridional mean-wind acceleration to eastward in both northern and southern hemispheres. It will be interesting to compare these wave-propagation directions and mean-wind variations near the mesopause region in the future.

Since the propagation directions of waves in the mesosphere (Fig. 3(a)) and in the thermosphere (Fig. 3(b)) are quite dissimilar at all stations, it is not likely that all the observed gravity waves in the mesosphere directly penetrate into the thermosphere. At midlatitudes, we should also consider the ionospheric Perkins instability, which may pick up only a favorite phase-front direction (NW-SE) from seeding gravity waves. Vadas and Fritts $(2005,2006)$ derived a gravity-wave anelastic dispersion relation that included molecular viscosity and thermal diffusivity to determine gravity-wave dissipation altitudes within the thermosphere. The dissipation altitude varies depending on intrinsic frequency and horizontal and vertical scales, as well 
as on the temperature in the thermosphere. A hot thermosphere enables deeper penetration than a cool thermosphere, and gravity waves with larger vertical wavelengths penetrate to higher altitudes than those with smaller vertical wavelengths. To investigate Vadas and Fritts (2005, 2006)'s prediction on the penetration of gravity waves from the mesopause region to the thermosphere, comparison of mesospheric and thermospheric airglow images, like that shown in Figs. 4-6, is a useful procedure. The subtraction of 1-h running averages or some sort of spatial/temporal filtering would be essential to figure out large-scale gravity waves in the relatively limited field of view of the mesospheric airglow images. For such purposes, 557.7-nm emission should be used with caution due to contamination of thermospheric green-line emissions, as shown in Fig. 4, particularly at equatorial latitudes. At other latitudes, Shepherd et al. (1997) stated that this contamination is virtually non-existent, according to the observation by the WIND imaging interferometer (WINDII) on board the upper atmosphere research satellite (UARS). With these considerations and careful data analysis, airglow imaging observation would be a useful tool to investigate the acceleration of mean wind or secondary-wave generation by gravity waves in the mesopause region and penetration of the waves into the thermosphere.

Acknowledgments. We thank Y. Katoh, M. Satoh, and T. Katoh of the Solar-Terrestrial Environment Laboratory, Nagoya University, for their helpful support of the development and operation of the OMTIs. The observation at Shigaraki was carried out in collaboration with the Research Institute for Sustainable Humanosphere, Kyoto University. The observation at Kototabang was carried out in collaboration with the Research Institute for Sustainable $\mathrm{Hu}-$ manosphere (RISH), Kyoto University, Japan, and the National Institute of Aeronautical and Space Science (LAPAN), Indonesia. The measurements of OMTIs have been supported by Grantsin-Aid for Scientific Research $(11440145,13573006,13136201$, 16403007, 18403011, 19403010, 20244080, and Priority Area 764) and Dynamics of the Sun-Earth-Life Interactive System (No.G-4, the 21st Century COE Program) of the Ministry of Education, Culture, Sports, Science and Technology of Japan.

\section{References}

Ejiri, M. K., K. Shiokawa, T. Ogawa, K. Igarashi, T. Nakamura, and T. Tsuda, Statistical study of short-period gravity waves in $\mathrm{OH}$ and $\mathrm{OI}$ nightglow images at two separated sites, J. Geophys. Res., 108(D21), 4679, doi:10.1029/2002JD002795, 2003.

Fritts, D. C., S. L. Vadas, and Y. Yamada, An estimate of strong local body forcing and gravity wave radiation based on $\mathrm{OH}$ airglow and meteor radar observations, Geophys. Res. Lett., 29, 1429, doi: 10.1029/2001GL013753, 2002.

Fukao, S., M. C. Kelley, T. Shirakawa, T. Takami, M. Yamamoto, T. Tsuda, and S. Kato, Turbulent upwelling of the mid-latitude ionosphere 1. Observational results by the MU radar, J. Geophys. Res., 96, 37253746, 1991.

Hosokawa, K., K. Shiokawa, Y. Otsuka, A. Nakajima, T. Ogawa, and J. D. Kelly, Estimating drift velocity of polar cap patches with allsky airglow imager at Resolute Bay, Canada, Geophys. Res. Lett., 33 , L15111, doi:10.1029/2006GL026916, 2006.

Kelley, M. C. and S. Fukao, Turbulent upwelling of the mid-latitude ionosphere 2. Theoretical framework, J. Geophys. Res., 96, 3747-3753, 1991.

Kelley, M. C. and J. J. Makela, Resolution of the Discrepancy between Experiment and Theory of Midlatitude F-Region Structures, Geophys. Res. Lett., 28(13), 2589-2592, 2001.

Kotake, N., Y. Otsuka, T. Tsugawa, T. Ogawa, and A. Saito, Climatological study of GPS total electron content variations caused by medium- scale traveling ionospheric disturbances, J. Geophys. Res., 111, A04306, doi:10.1029/2005JA011418, 2006.

Kubota, M., K. Shiokawa, M. K. Ejiri, Y. Otsuka, T. Ogawa, T. Sakanoi, H. Fukunishi, M. Yamamoto, S. Fukao, and A. Saito, Traveling ionospheric disturbances observed in the OI 630-nm nightglow images over Japan by using a multi-point imager network during the FRONT campaign, Geophys. Res. Lett., 24, 4037-4040, 2000.

Mendillo, M., J. Baumgardner, D. Nottingham, J. Aarons, B. Reinisch, J. Scali, and M. Kelley, Investigations of thermospheric-ionospheric dynamics with $6300 \mathrm{~A}$ images from the Arecibo Observatory, J. Geophys. Res., 102, 7331-7343, 1997.

Miller, C. A., W. E. Swartz, M. C. Kelley, M. Mendillo, D. Nottingham, J. Scali, and B. Reinisch, Electrodynamics of midlatitude spread $F$, 1. Observations of unstable, gravity wave-induced ionospheric electric fields at tropical latitudes, J. Geophys. Res., 102, 11,521-11,532, 1997.

Nakamura, T., A. Higashikawa, T. Tsuda, and Y. Matsushita, Seasonal variations of gravity wave structures in $\mathrm{OH}$ airglow with a CCD imager at Shigaraki, Earth Planets Space, 51, 897-906, 1999.

Nakamura, T., T. Aono, T. Tsuda, A. G. Admiranto, E. Achmad, and Suranto, Mesospheric gravity waves over a tropical convective region observed by $\mathrm{OH}$ airglow imaging in Indonesia, Geophys. Res. Lett., 30(17), 1882, doi:10.1029/2003GL017619, 2003.

Ogawa, T., E. Sagawa, Y. Otsuka, K. Shiokawa, T. J. Immel, S. B. Mende, and P. Wilkinson, Simultaneous ground- and satellite-based airglow observations of geomagnetic conjugate plasma bubbles in the equatorial anomaly, Earth Planets Space, 57, 385-392, 2005.

Ogawa, T., Y. Otsuka, K. Shiokawa, T. Tsugawa, A. Saito, K. Hoshinoo, K. Matunaga, M. Kubota, and M. Ishii, Medium-scale traveling ionospheric disturbances and plasma bubbles observed by an all-sky airglow imager at Yonaguni, Japan, Terr. Atmos. Ocean Sci., 2008 (in press).

Otsuka, Y., K. Shiokawa, T. Ogawa, and P. Wilkinson, Geomagnetic conjugate observations of equatorial airglow depletions, Geophys. Res. Lett., 29(15), 43-1-4, 10.1029/2002GL015347, 2002.

Otsuka, Y., K. Shiokawa, T. Ogawa, and P. Wilkinson, Geomagnetic conjugate observations of medium-scale traveling ionospheric disturbances at midlatitude using all-sky airglow imagers, Geophys. Res. Lett., L15803, doi:10.1029/2004GL020262, 2004.

Otsuka, Y., K. Shiokawa, and T. Ogawa, Equatorial ionospheric scintillations and zonal irregularity drifts observed with closely-spaced GPS receivers in Indonesia, J. Meteor. Soc. Jpn., 84A, 343-351, 2006.

Perkins, F., Spread F and ionospheric currents, J. Geophys. Res., 78, 218226, 1973.

Saito, A., M. Nishimura, M. Yamamoto, M. Kubota, K. Shiokawa, Y. Otsuka, T. Tsugawa, S. Fukao, T. Ogawa, M. Ishii, T. Sakanoi, and S. Miyazaki, Traveling ionospheric disturbances detected in the FRONT campaign, Geophys. Res. Lett., 28, 689-692, 2001.

Sakaguchi, K., K. Shiokawa, A. Ieda, Y. Miyoshi, Y. Otsuka, T. Ogawa, M. Connors, E. F. Donovan, and F. J. Rich, Simultaneous ground and satellite observations of an isolated proton arc at subauroral latitudes, $J$. Geophys. Res., 112, A04202, doi:10.1029/2006JA012135, 2007.

Shepherd, G. G., R. G. Roble, C. McLandress, and W. E. Ward, WINDII observations of the $558 \mathrm{~nm}$ emission in the lower thermosphere: The influence of dynamics on composition, J. Atmos. Sol.-Terr. Phys., 59(6), 655-667, 1997.

Shiokawa, K., Y. Katoh, M. Satoh, M. K. Ejiri, T. Ogawa, T. Nakamura, T. Tsuda, and R. H. Wiens, Development of optical mesosphere thermosphere imagers (OMTI), Earth Planets Space, 51, 887-896, 1999.

Shiokawa, K., Y. Katoh, M. Satoh, M. K. Ejiri, and T. Ogawa, Integratingsphere calibration of all-sky cameras for nightglow measurements, $A d v$. Space Sci., 26, 1025-1028, 2000a.

Shiokawa, K., M. K. Ejiri, Y. Otsuka, T. Ogawa, M. Kubota, K. Igarashi, A. Saito, and T. Nakamura, Multi-point observations of short-period mesospheric gravity waves over Japan during the FRONT campaign, Geophys. Res. Lett., 24, 4057-4060, 2000b.

Shiokawa, K., Y. Otsuka, M. K. Ejiri, Y. Sahai, T. Kadota, C. Ihara, T. Ogawa, K. Igarashi, S. Miyazaki, and A. Saito, Imaging observations of the equatorward limit of midlatitude traveling ionospheric disturbances, Earth Planets Space, 54, 57-62, 2002.

Shiokawa, K., C. Ihara, Y. Otsuka, and T. Ogawa, Statistical study of nighttime medium-scale traveling ionospheric disturbances using midlatitude airglow images, J. Geophys. Res., 108(A1), 1052, doi:10.1029/2002JA009491, 2003a.

Shiokawa, K., Y. Otsuka, C. Ihara, T. Ogawa, and F. J. Rich, Ground and satellite observations of nighttime medium-scale traveling ionospheric disturbance at midlatitude, J. Geophys. Res., 108(A4), 1145, doi:10.1029/2002JA009639, 2003b. 
Shiokawa, K., Y. Otsuka, T. Ogawa, and P. Wilkinson, Time evolution of high-altitude plasma bubbles imaged at geomagnetic conjugate points, Ann. Geophys., 22, 3137-3143, 2004.

Shiokawa, K., Y. Otsuka, T. Tsugawa, T. Ogawa, A. Saito, K. Ohshima, M. Kubota, T. Maruyama, T. Nakamura, M. Yamamoto, and P. Wilkinson, Geomagnetic conjugate observation of nighttime medium- and largescale traveling ionospheric disturbances: FRONT3 campaign, J. Geophys. Res., 110, A05303, doi:10.1029/2004JA010845, 2005.

Shiokawa, K., Y. Otsuka, and T. Ogawa, Quasiperiodic southward moving waves in 630-nm airglow images in the equatorial thermosphere, $J$. Geophys. Res., 111, A06301, doi:10.1029/2005JA011406, 2006.

Sobral, J. H. A., H. Takahashi, M. A. Abdu, P. Muralikrishna, Y. Sahai, C. J. Zamlutti, E. R. DE Paura, and P. P. Batista, Determination of the quenching rate of the $\mathrm{O}(1 \mathrm{D})$ by $\mathrm{O}(3 \mathrm{P})$ from rocket-borne optical $(630$ $\mathrm{nm}$ ) and electron density data, J. Geophys. Res., 98, 7791-7798, 1993.

Suzuki, S., Study of mesospheric gravity wave dynamics based on airglow imaging observations at middle and low latitudes, Ph.D. thesis, Nagoya University, 2007.

Suzuki, S., K. Shiokawa, Y. Otsuka, T. Ogawa, and P. Wilkinson, Statistical characteristics of gravity waves observed by an allsky imager at Darwin, Australia, J. Geophys. Res., 109, D20S07, doi:10.1029/2003JD004336, 2004.

Suzuki, S., K. Shiokawa, Y. Otsuka, T. Ogawa, M. Kubota, M. Tsutsumi, T. Nakamura, and D. C. Fritts, Gravity wave momentum flux in the upper mesosphere derived from $\mathrm{OH}$ airglow imaging measurements, Earth Planets Space, 59, 421-428, 2007.

Taylor, M. J., M. B. Bishop, and V. Taylor, All-sky measurements of short period waves imaged in the $\mathrm{OI}(557.7 \mathrm{~nm}), \mathrm{Na}(589.2 \mathrm{~nm})$ and near infrared $\mathrm{OH}$ and $\mathrm{O}_{2}(0,1)$ nightglow emissions during the ALOHA-93 campaign, Geophys. Res. Lett., 22, 2833-2836, 1995.

Tsuda, T., Y. Murayama, M. Yamamoto, S. Kato, and S. Fukao, Seasonal variation of momentum flux in the mesosphere observed with the MU radar, Geophys. Res. Lett., 17(6), 725-728, 1990.

Vadas, S. L., Horizontal and vertical propagation and dissipation of gravity waves in the thermosphere from lower atmospheric and thermospheric sources, J. Geophys. Res., 112, A06305, doi:10.1029/2006JA011845, 2007.

Vadas, S. L and D. C. Fritts, Thermospheric responses to gravity waves: Influences of increasing viscosity and thermal diffusivity, J. Geophys. Res., 110, D15103, doi:10.1029/2004JD005574, 2005.

Vadas, S. L. and D. C. Fritts, Influence of solar variability on gravity wave structure and dissipation in the thermosphere from tropospheric convection, J. Geophys. Res., 111, A10S12, doi:10.1029/2005JA011510, 2006.

Vadas, S. L., D. C. Fritts, and M. J. Alexander, Mechanism for the generation of secondary waves in wave breaking regions, J. Atmos. Sci., 60 , 194-214, 2003.

Vincent, R. A., Gravity-wave motions in the mesosphere, J. Atmos. Terr. Phys., 46, 119-128, 1984.

Walterscheid, R. L., J. H. Hecht, R. A. Vincent, I. M. Reid, J. Woithe, and M. P. Hickey, Analysis and interpretation of airglow and radar observations of quasi-monochromatic gravity waves in the upper mesosphere and lower thermosphere over Adelaide, Australia $\left(35^{\circ} \mathrm{S}, 138^{\circ} \mathrm{E}\right), J$. Atmos. Terr. Phys., 61, 461-478, 1999.

K. Shiokawa (e-mail: shiokawa@stelab.nagoya-u.ac.jp), Y. Otsuka, and T. Ogawa 